\title{
Klinik Schloss Mammern, eine der ältesten Privatkliniken der Schweiz
}

\author{
R. Fleisch-Silvestri, A. Fleisch Marx
}

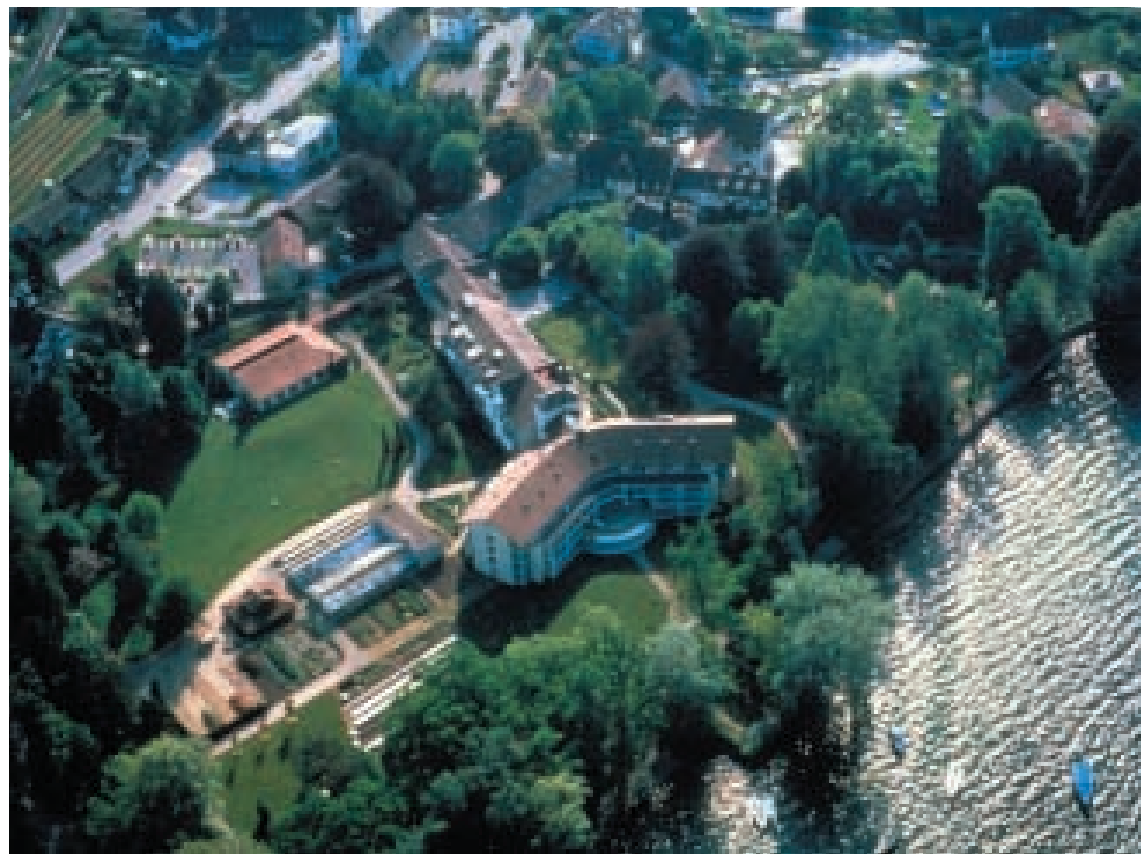

Gefässdoppler, Gastro- und Kolonoskopie sowie Densitometrie. Zwei Überwachungszimmer mit drei Betten stehen für Notfälle bereit.

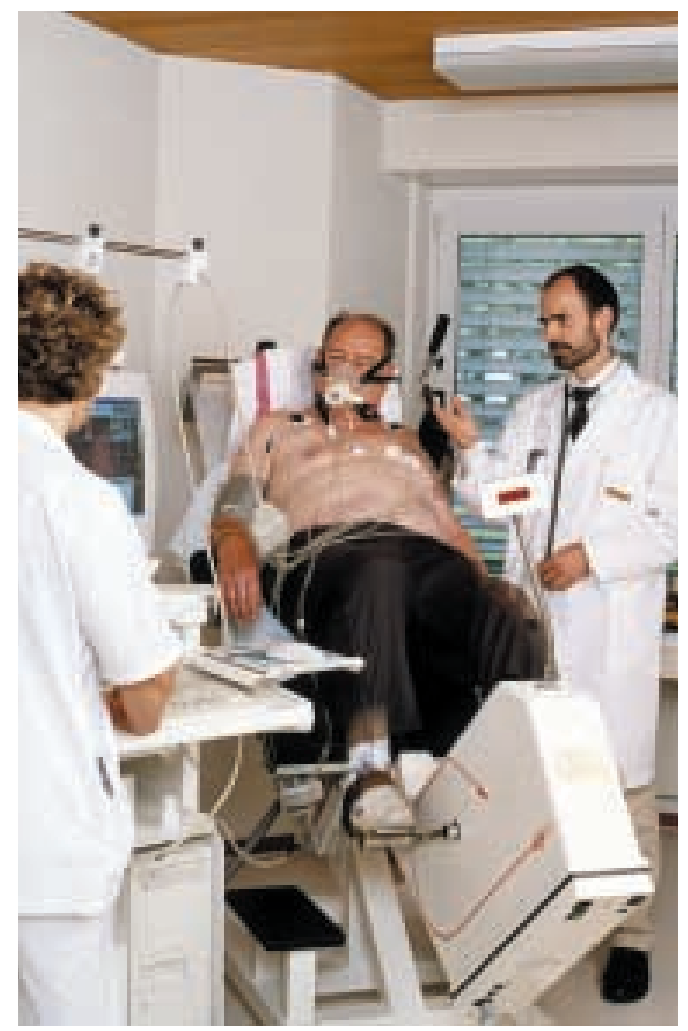

spital für Innere Medizin mit integrierter Rehabilitation. Sie umfasst 125 Betten und beschäftigt 200 Mitarbeiter.

Im Akutbereich stehen Abklärungen und Therapien aus dem ganzen Spektrum der inneren Medizin im Vordergrund. Sechs festangestellte Ärzte mit verschiedenen Facharzttiteln (Innere Medizin, Kardiologie, Rheumatologie, Rehabilitation und physikalische Medizin) und Fähigkeitsausweisen und zurzeit eine Assistenzärztin bilden das ärztliche Team. Zusätzlich stehen uns Konsiliarärzte auf folgenden Gebieten zur Verfügung: Neurologie, Psychiatrie, Psychosomatik, Radiologie, Orthopädie, Pneumologie und Dermatologie. Im Pflegebereich arbeiten etwa 45 Mitarbeiter/innen.

Die medizinische Abteilung verfügt über die Einrichtungen eines mittelgrossen Spitales, unter anderem Labor, Röntgen, Sonographie, EKG, Ergometrie, 24-Stunden-BD-Messung, 24-Stunden-EKG, Farbdopplerechokardiographie, transösophageale Echokardiographie, Stress-Echokardiographie, Schlafoximetrie, Spiroergometrie,

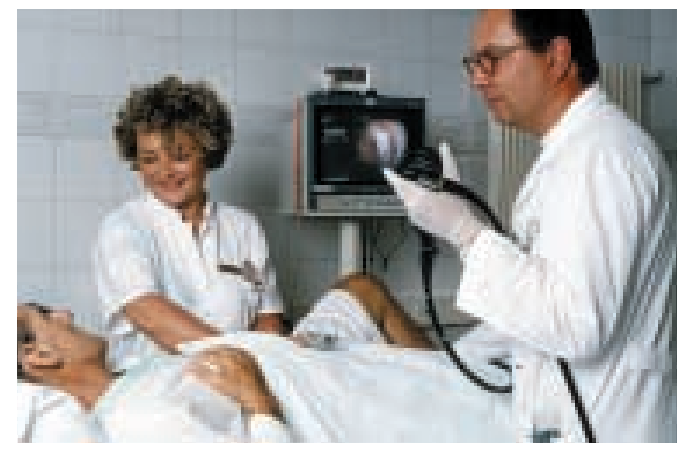

In der Rehabilitation liegt das Schwergewicht in der kardialen, rheumatologisch-orthopädischen, internistischen und allgemeinen postoperativen Rehabilitation. Die Patienten sind in der Regel multimorbid. Die Wiedererlangung bzw. die Verbesserung der Selbständigkeit, die Integration ins soziale und berufliche Umfeld und die Sekundärprophylaxe stehen im Vordergrund.

Dr. med. Annemarie Fleisch Marx Klinik Schloss Mammern CH-8265 Mammern 
Die rehabilitative Pflege und der körperliche Aufbau individuell oder in kleinen Gruppen sind vorrangig. Die kardiale Rehabilitation wird gemäss Richtlinien der Schweizerischen Arbeitsgruppe für kardiale Rehabilitation (SAKR) durchgeführt. Instruktionen über risikohaftes Verhalten und gesunde Lebensführung sind integraler Bestandteil der Rehabilitation.

Wir beschäftigen 14 Physiotherapeuten, 4 Sporttherapeuten, 2 Ergotherapeutinnen und 1 Logopädin. Die Behandlung ist patientenbezogen und die Individualität und Lebenssituation der erkrankten Person wird berücksichtigt.

Die Kombination Akutspital und Rehabilitation hat sich aus der Patientenkonstellation heraus entwickelt. Schon immer wurden eher ältere Patienten mit verschiedenen medizinischen Problemen zugewiesen, sogenannte multimorbide Patienten. Dank der Spitalinfrastruktur können nichtchirurgische Komplikationen der Rehabilitationspatienten in der Klinik behandelt werden. Bei Intensivstationnotwendigkeit wird eine enge und konstruktive Zusammenarbeit mit den zwei Thurgauer Kantonsspitälern gepflegt.

Ambulante Medizin: Auf Zuweisung werden spezialärztliche Abklärungen durchgeführt. Die Klinik Schloss Mammern ist für den Kanton Thurgau die offizielle ambulante kardiologische Rehabilitationsstätte.

Die Klinik hat einen KVG-konformen Gesamtversicherungsvertrag für Thurgauer Patienten. Im KVG-Bereich bestehen mit allen Krankenkassen Zusatzversicherungsverträge in der Halb- und Privatabteilung.

Das Durchschnittsalter der Patienten ist 69 Jahre. 85 \% der Patienten sind ausserkantonal, $10 \%$ davon stammen aus dem Ausland.

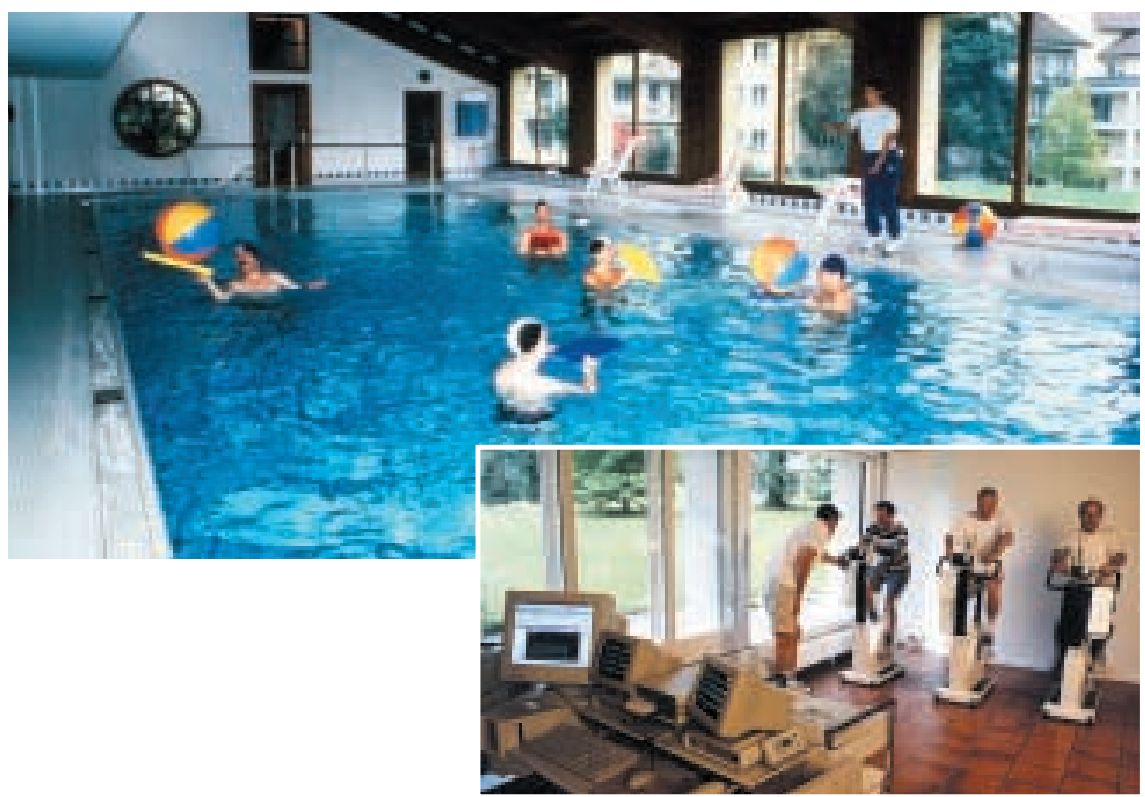

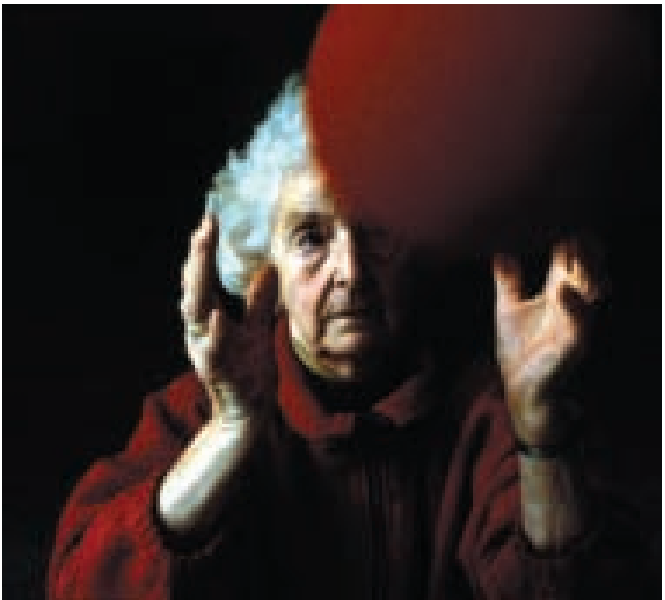
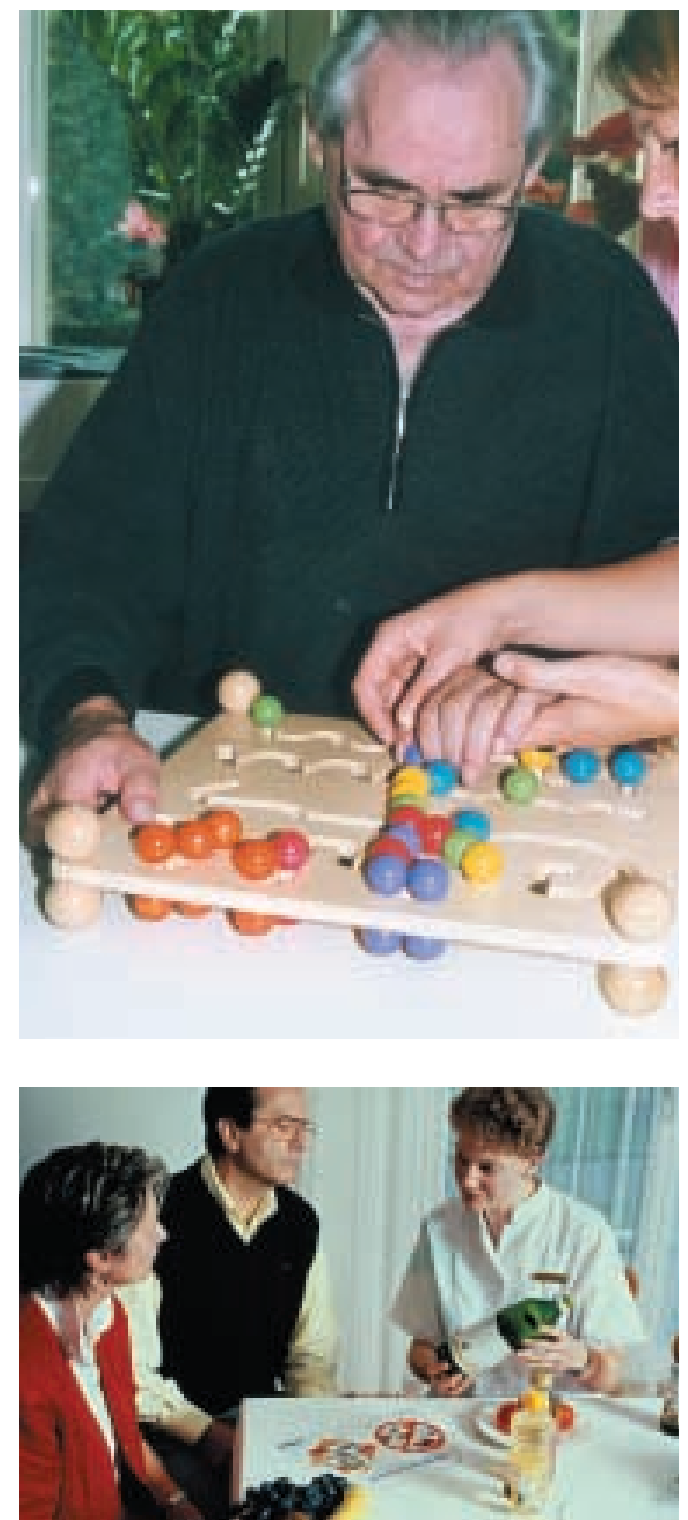
Bei hohem Qualitätsbewusstsein ist es eine Selbstverständlichkeit, dass die Vorgaben der ALVR in allen angebotenen Rehabilitationsrichtungen eingehalten werden. Die Klinik Schloss Mammern ist Weiterbildungsstätte für Kardiologie, Innere Medizin und Physikalische Medizin und Rehabilitation.

Bekannt ist die Klinik auch für ihre vorzügliche Hotellerie. Eine schöne Umgebung, eine exzellente Küche und eine freundliche und zuvorkommende Betreuung fördern den Genesungsprozess.

Die Klinik Schloss Mammern liegt inmitten eines prachtvollen, $90000 \mathrm{~m}^{2}$ grossen Parks direkt am Untersee.
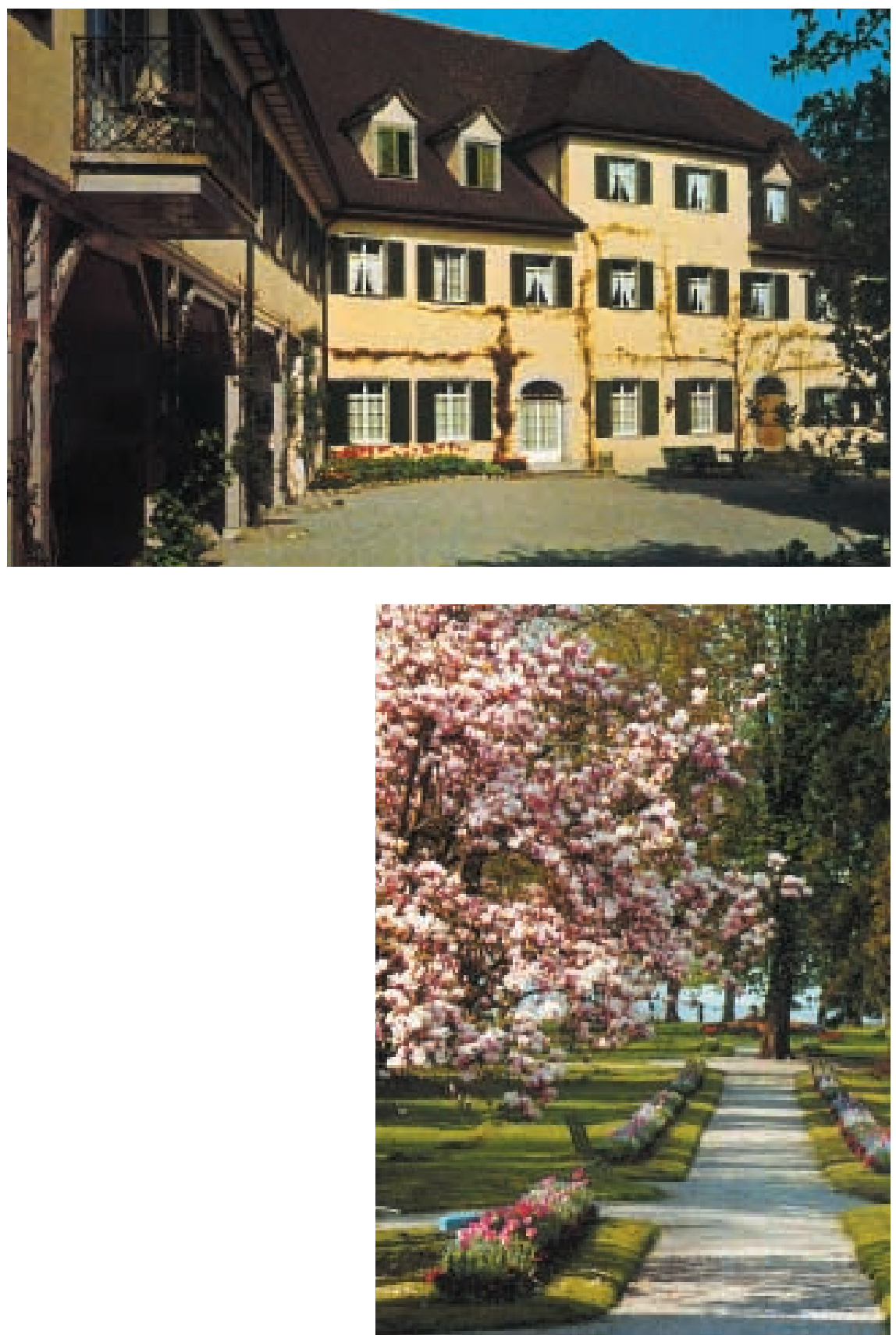

\section{Die Klinik Schloss Mammern in der Vergangenheit}

Die wechselhafte Geschichte der Klinik Schloss Mammern erstreckt sich über eine Zeitspanne von 381 Jahren.

1621 bauten Ritter aus dem Geschlechte von Roll das «Neue Schloss» auf den Mauern eines befestigten Amtshauses. In der Folge wechselten die Besitzer mehrmals. Von 1686 bis 1838 residierten die Äbte vom Kloster Rheinau in der Herrschaft Mammern.

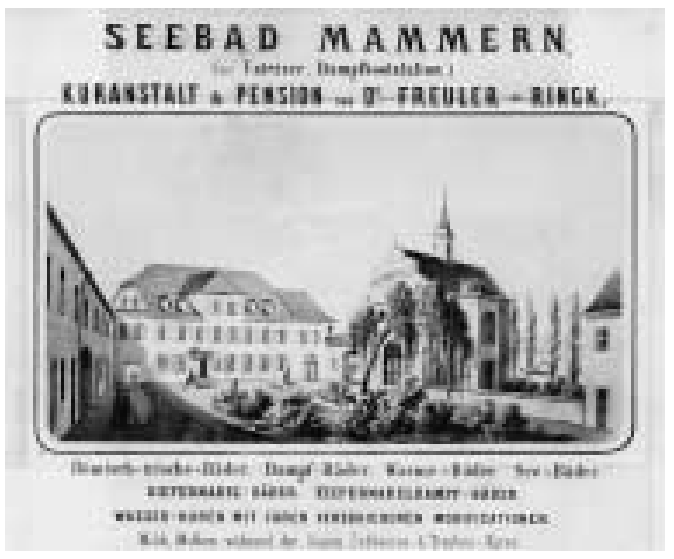

1866 wurde in den historischen Gebäuden durch Dr. Freuler eine Wasserheilanstalt gegründet. Seit über 130 Jahren werden somit in der heutigen Klinik Schloss Mammern Patienten betreut.

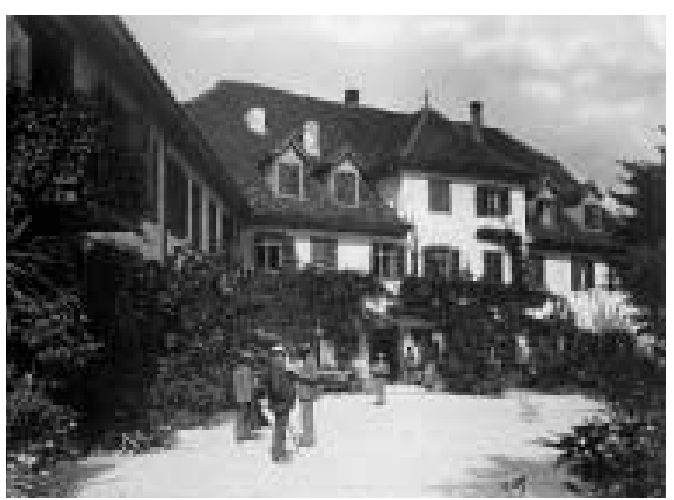

Seit 1889 ist das Schicksal der heutigen Klinik Schloss Mammern eng mit der Familie UllmannFleisch verbunden. Dr. Oscar Ullmann kaufte die Wasserheilanstalt zum damals horrenden Preis von Fr. 300 000.-.

Ein das ganze Jahr geöffnetes Sanatorium für Nervenkranke und Fälle von Neurasthenie, Hysterie und Hypochondrie wurde eingeführt. Die bisherige Wasserheilanstalt wurde daneben weitergeführt. 


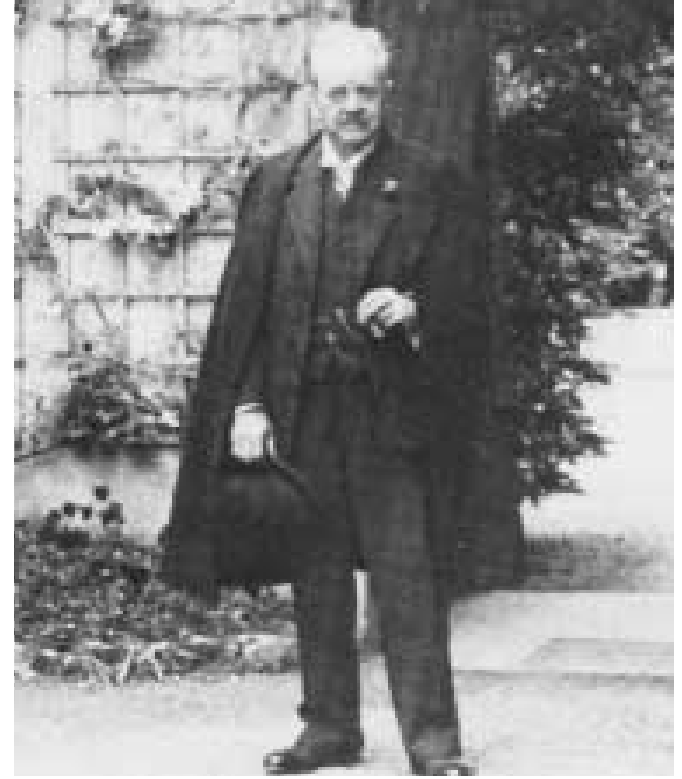

Dr. Ullmann war ein glänzender Psychotherapeut und hatte grosses Geschick in der Führung seiner Patienten. Die Anstalt prosperierte unter seiner Führung zu grosser Blüte. Es strömten $\mathrm{Pa}$ tienten aus aller Herren Länder zu Dr. Ullmann, die Pflegetage stiegen in den ersten Jahren des 20. Jahrhunderts auf $20000 / \mathrm{Jahr}$ an.

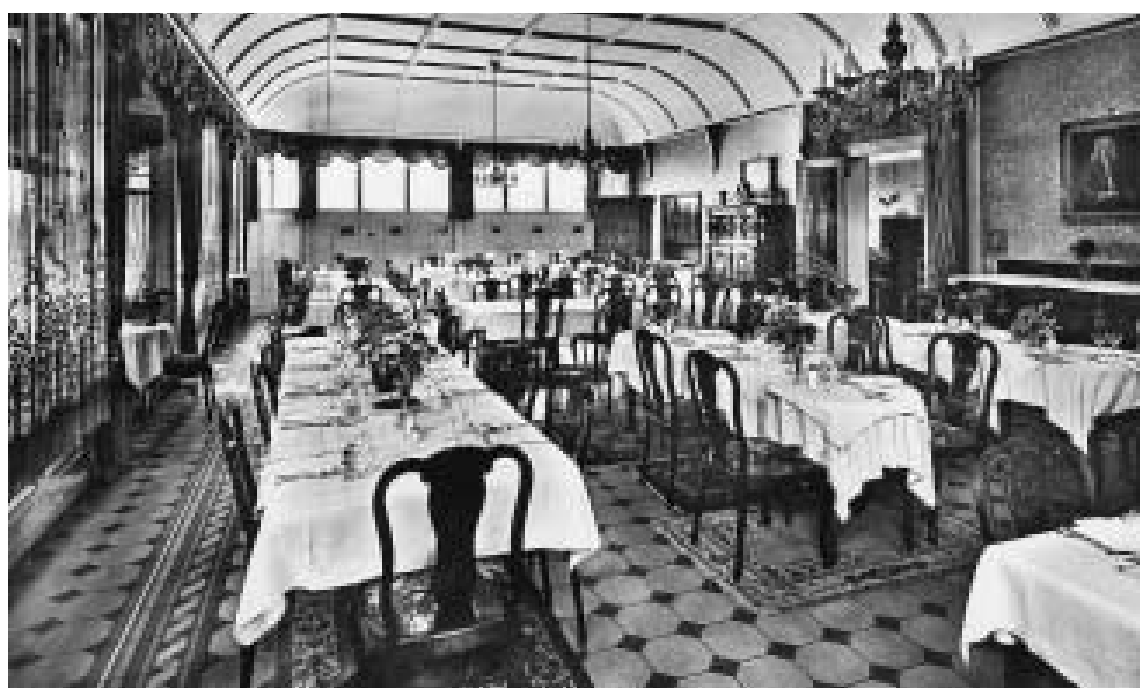

Während der Blütezeit wurde regelmässig in bauliche und medizinische Neuerungen investiert: Noch vor Ende des Jahrhunderts Einführung des elektrischen Lichtes, Bau eines neuen Gästehauses, Landkauf und Rückkauf der Schlosskapelle zum Preis von Fr. 35 000.-, Einführung von Medikamenten wie Arsenspritzen und Chloraldurat, BD-Messung.

Es erfolgte die Umbenennung der Wasserheilanstalt in Kuranstalt Mammern.

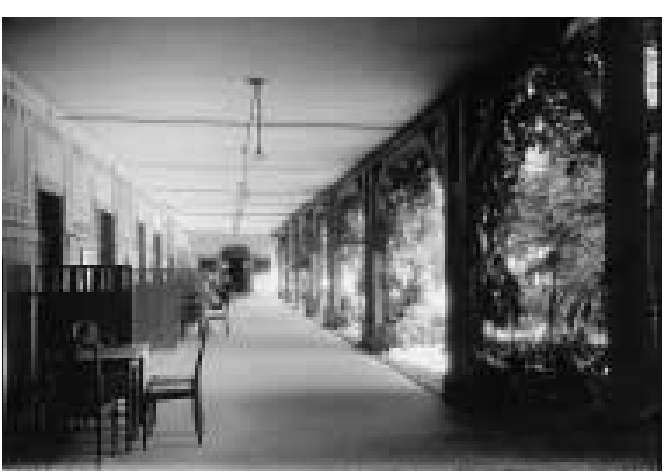

Der Ausbruch des 1. Weltkrieges am 1. August 1914 versetzte dem florierenden Unternehmen jedoch einen schweren Schlag. Am 2. August 1914 reisten 100 Kurgäste gleichzeitig ab. Mit diesem Tag war die erste grosse Blütezeit von Mammern vorbei.

Von 1926 bis 1944 führte Waldemar Ullmann, der Sohn von Oscar Ullmann, den Betrieb. Dank seiner Ausbildung in Innerer Medizin bekam das Haus einen mehr internistischen Charakter. Anschaffung eines Röntgenapparates und Einführung der aktuellen Pharmakopoe. Der Prospekt wurde weniger blumig und der Indikationskatalog umfasste die üblichen internistischen Erkrankungen. In den ersten Jahren nahm die Zahl der Verpflegungstage wieder stetig zu.

Die Weltkrise 1929/33 und der 2. Weltkrieg führten jedoch zu einem zunehmenden Niedergang des ehemals prosperierenden Betriebes. Schwere finanzielle Sorgen drückten den Besitzer. Zudem hatte Dr. Ullmann mit der neuen Medizin nicht Schritt gehalten und sich trotz seines guten Rufes als Herzspezialist nicht weiter in die Materie eingearbeitet. Es war kein Geld mehr für Reparaturen und Unterhalt vorhanden, die Gebäude verwahrlosten immer mehr, die Einrichtungen waren veraltet und Mammern stand im Ruf, alt und verlottert zu sein.

Am 14. September 1944 wurde Dr. Waldemar Ullmann durch einen vorbestraften Hilfsgärtner erschossen. Der Betrieb stand verwaist da.

Um den Fortbestand des Familienbetriebes zu gewährleisten, übernahm Prof. Alfred Fleisch, Ehegatte von Ilse Ullmann, den Betrieb. Er war als Professor für Physiologie und physiologische Chemie an der Universität Lausanne tätig und führte den Betrieb administrativ vom Welschland aus. In der Klinik war ein Arzt aus dem Nachbardorf im Teilzeitpensum tätig.

Die Gebäude waren bei der Übernahme in einem jämmerlichen Zustand. Um einen gewissen Standard zu erreichen, wurde jedes Jahr in die bauliche Substanz investiert. 


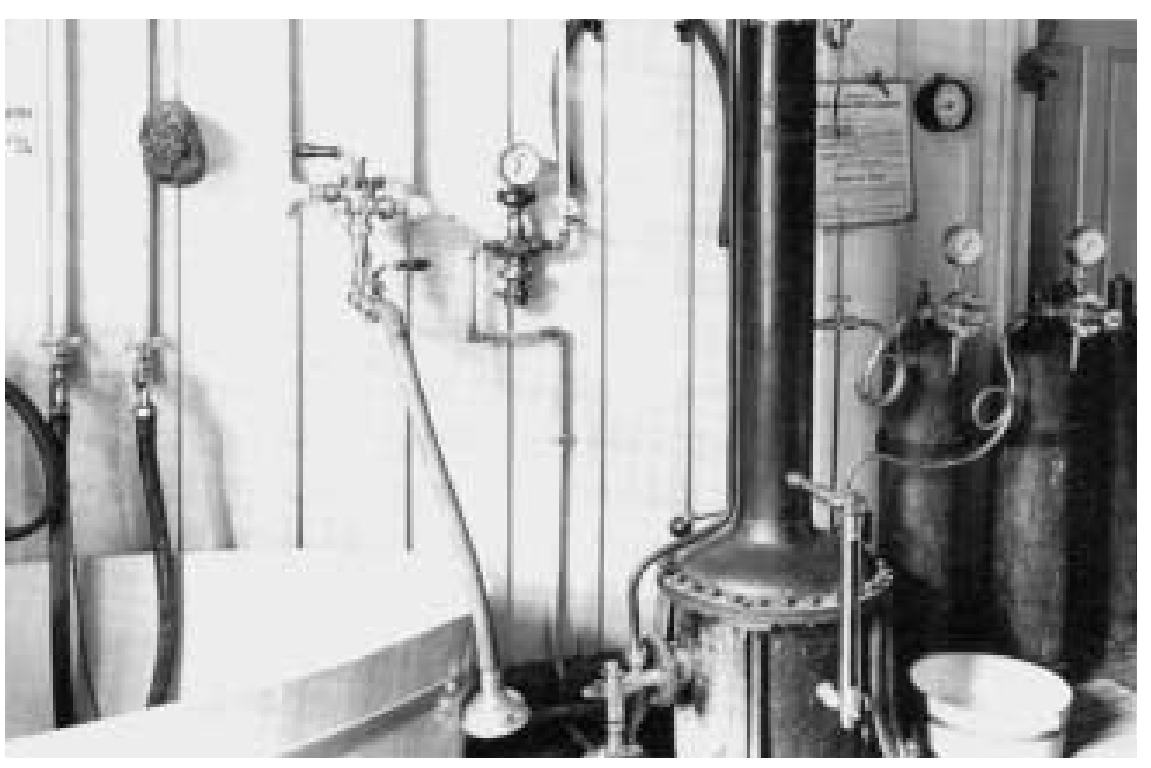

Bis 1957 war das Ergebnis der Klinik jedoch tiefrot. Die Deckung des Defizites erfolgte aus dem privaten Einkommen von Prof. Fleisch.

Seit 1958 leitet Dr. Alfred Oscar Fleisch gemeinsam mit seiner Ehegattin Edith FleischZehnder sehr erfolgreich die Geschicke der Klinik Schloss Mammern. Nach seiner internistischen und kardiologischen Ausbildung im Inund Ausland trat er am 1. Mai 1958 seine neue Wirkungsstätte an. Die Gästezahl war zu Beginn deprimierend tief: Bei etwa 60 Angestellten waren ganze 10 Gäste im Haus.

Die medizinische Abteilung bestand aus zwei winzig kleinen Sprechzimmern, einem schwarz tapezierten Röntgenzimmer mit einem 35 Jahre alten Durchleuchtungsapparat, einem kleinen Labor mit Mikroskop und 30 Jahre alten Medikamenten.

Die Patienten waren meistens erholungssuchende Gesunde.

Dank grossem ärztlichem, geschäftlichem und politischem Geschick und einem enormen Arbeitseinsatz entwickelte sich die Klinik während seiner jetzt 44jährigen Tätigkeit von einem defizitären Kurhaus zum heutigen florierenden «Akutspital mit integrierter Rehabilitation».

Im Laufe der Jahre nahm der klinische Charakter des Hauses stark zu. Aus dem Kurhaus mit Erholungssuchenden wurde progressiv ein Sanatorium und später eine Klinik. Anfänglich konnten keine Spitalpatienten aufgenommen werden, da nicht genügend Pflegepersonal zur Verfügung stand. 1965 beschäftigte der Betrieb 5 Schwestern, 2 Laborantinnen, 1 Röntgenassistentin, 1 Diätassistentin, 2 Physiotherapeuten und 2 vollamtliche Ärzte. Heute sind etwa 80 Angestellte im medizinischen Sektor tätig.
1966 wurde im Rahmen der medizinischen Veränderungen der Name «Kuranstalt» in «Klinik Schloss Mammern» geändert.

Die Verpflegungstage stiegen wieder an bis auf 35000 .

Bei zunehmender Gästezahl und höheren Ansprüchen in der Medizin und Hotellerie mussten die baulichen und medizinischen Anlagen ständig ausgebaut werden.

Über die Jahre erfolgten insgesamt drei grosse Erweiterungsbauten zur Beherbergung der Patienten und der komplizierter werdenden Infrastruktur. 1984 Bau eines Physiotherapiezentrums, 1980-93 Restauration der Barockkapelle (Baujahr 1749).

1995 zog sich Dr. A. O. Fleisch aus dem medizinischen Tagesgeschäft zurück. Von 1994 bis 1996 war PD Dr. Horber als medizinischer Chefarzt tätig. Seit 1997 wird die Klinik in der 4. Generation der Gründerfamilie durch Dr. R. Fleisch-Silvestri und Dr. A. Fleisch Marx im Co-Chefärztinnen-System geführt.

\section{Die Privatspitäler und das KVG}

$20 \%$ der Patienten in der Schweiz werden in Privatspitälern betreut. Die neun Thurgauer Privatspitäler vom Verband der Ostschweizer Privatkliniken OPK betreuten auch im Jahr 2001 $1 / 3$ der stationären Patienten, die sich im Kanton Thurgau behandeln liessen.

Die Privatspitäler des Kantons Thurgau erfüllen somit eine wichtige Aufgabe in der Betreuung der Patienten, als Arbeitgeber und als Wirtschaftsfaktor.

Kennzahlen im Jahr 2001 in Kürze: 9 Kliniken, 642 Betten, 9373 behandelte Patienten, 195518 Pflegetage (38\% Kantonseinwohner, 62\% andere Kantone und Ausland), 1150 beschäftigte Personen, Personalaufwand 70 Mio. Franken, Sachaufwand 32 Mio. Franken.

Seit 2002 hat sich die OPK auf die ganze Ostschweiz vergrössert. Heute zählt sie zu den neun Thurgauer Gründungskliniken weitere vier Kliniken im Appenzell, drei Kliniken im St. Gallischen und eine im Kanton Schaffhausen.

«Privat» bedeutet nicht «Privatpatient», sondern «private Trägerschaft» ohne Subventionen vom Bund oder Kanton. Entgegen der landläufigen Meinung betreuen Privatspitäler somit nicht ausschliesslich Privatpatienten, sondern führen auch Allgemeinabteilungen für grundversicherte Patienten. Im Kanton Thurgau bieten die privaten Leistungserbringer erstklassige medizinische Diagnostik und entsprechende Behand- 
lungen auch für Grundversicherte in praktisch allen Fachbereichen an.

Durch die Einführung des KVG 1996 (nach der knappen Abstimmung 1994 mit 51\% JaStimmen) wurde die Lage der Privatspitäler im Gesundheitsmarkt Schweiz deutlich schlechter. Ein neues Gesetz mit 35 Seiten und 107 Artikeln erwähnt die Privatspitäler, die in der Schweiz $20 \%$ der medizinischen Versorgung garantieren, nur gerade mit folgendem Teilartikel: Art. 39, Abs. 1, Punkt d «private Trägerschaften sind angemessen in die Planung einzubeziehen». Die juristischen Folgen liessen nicht lange auf sich warten. Ein Jahr später wurde schon eine parlamentarische Initiative für die «Freie Arzt- und Spitalwahl» in Bern eingereicht. 1998 lagen beim Bund seit der Einführung des Gesetzes zusätzlich 179 Rekurse gegen das KVG vor. Die 2. Teilrevision des KVG - die Spitalfinanzierung - ist seit 1999 in Vernehmlassung.

Für die Klinik Schloss Mammern mit ihrem breiten medizinischen Angebot und dem hohen ausserkantonalen Anteil an Patienten waren die politischen Entwicklungen schwierig. Dank intensivem personellem und juristischem Einsatz wurde die Besonderheit der Strukturen jedoch durch den Kanton Thurgau anerkannt und das gesamte Leistungsspektrum auf die Spitalliste gesetzt.

Im Kanton Thurgau wurden auch die übrigen Privatspitäler korrekt berücksichtigt. Alle Privatspitäler des Kantons sind heute mit der definitiven und rechtsgültigen Spitalliste einverstanden.

Im Vergleich mit den öffentlichen Spitälern sind die Spiesse jedoch nach wie vor ungleich lang. Eine neue grosse Hürde für die Privatspitäler wurde mit dem Entscheid des EVG (Eidgenössisches Versicherungsgericht) im November 2001 aufgebaut. Dieser Entscheid bestätigt, das jeder Bürger grundversichert ist. Demzufolge muss nach KVG bei öffentlich-subventionierten Spitälern der Anteil von 50\% der Grundversi- cherungskosten bei einem Spitalaufenthalt vom Kanton übernommen werden, auch wenn der Patient auf der Halbprivat- oder Privatabteilung als Zusatzversicherter behandelt wird. Dieser EVG-Entscheid wurde mit Dringlichkeitsbeschluss am 21. Juni 2002 im Bundesgesetz verankert. Verheerend für die Privatspitäler ist, dass dieser Entscheid nur für öffentliche Spitäler Geltung hat. Dies führt dazu, dass die Kosten neu auch bei zusatzversicherten Patienten in Privatspitälern für die Krankenkassen automatisch um den sogenannten Sockelbetrag höher ausfallen als in öffentlichen Spitälern. Dies veranlasst die betriebswirtschaftlich geführten Krankenkassen verständlicherweise dazu, privatversicherte $\mathrm{Pa}$ tienten vermehrt in die öffentlichen Spitäler zu steuern. Auch werden Versicherungen mit eingeschränkter Wahl unter Ausschluss der Privatspitäler angeboten.

Die Assura Krankenkasse lancierte im Herbst 2002 mit Erfolg ein Referendum gegen den Dringlichkeitsbeschluss. Endziel ist, dass der Sockelbeitrag vom Kanton auch bei Privatspitälern geleistet werden soll. Obwohl finanziell die Spiesse dann zwar wieder gleich lang wären, wird dann die Quadratur des Kreises wieder bei der Spitalliste anlangen. Werden die Spitäler der OPK (Klinik Bellevuepark Venenklinik [TG], Herz- und Neuro-Zentrum Bodensee [TG], Klinik Schloss Mammern [TG], Klinik für Psychiatrie und Psychotherapie Littenheid [TG], Humaine Klinik Zihlschlacht [TG], Klinik Seeschau [TG], Klinik Bindersgarten [TG], Klinik Landhaus Aadorf [TG], Kneipphof Dussnang [TG], Klinik Gais [AR], Berit Paracelsus Klinik [AR], Hirslanden Klinik am Rosenberg [AR], Klinik Stephanshorn [SG], Klinik Marienfried [SG], Reha-Klinik Walenstadtberg [SG], Hirslanden Klinik Belair [SH]) noch auf der Spitalliste des Kantons erscheinen, wenn der Kanton bezahlen muss? Eine neue Welle von Bundesratsbeschwerden wird folgen. 